$$
\begin{aligned}
& =\sum_{2}^{x} \gamma(n)\left[\frac{\mathrm{I}}{n(\log n)^{2}}-\frac{\mathrm{I}}{(n+\mathrm{I})\{\log (n+\mathrm{I})\}^{2}}\right]+\frac{\gamma[x]}{([x]+\mathrm{I})\{\log ([x]+\mathrm{I})\}^{2}} \\
& =O \sum_{2}^{x} \frac{I}{V(\log n)^{2}}+O\left\{\frac{V_{x}^{x}}{(\log x)^{2}}\right\} \\
& =O\left\{\frac{\sqrt{x}}{(\log x)^{2}}\right\} \text {. }
\end{aligned}
$$

From (5. 83) and (5. 84) it follows that

$$
f(x)-L i x-\frac{\psi(x)-x}{\log x}=O\left\{\frac{V x}{(\log x)^{2}}\right\} ;
$$

and from (5. 85) and Theorem 5. 8 we deduce

Theorem 5. 81. We have

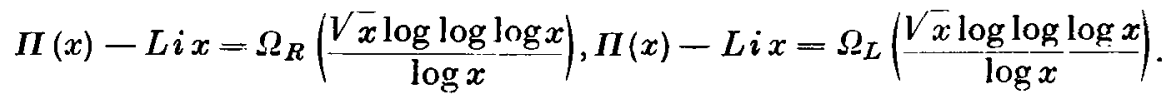

We refer in the introduction (I. 5) to the other important applications which may be made of the method of this section.

\title{
Additional Note.
}

While we have been engaged on the final correction of the proofs of this memoir, which was presented to the Acta Mathematica in the summer of Igr5, two very interesting notes by M. DE LA ValiÉE-Poussin entitled 'Sur les zéros de $\zeta(s)$ de RiemanN' have appeared in the Comptes Rendus (23 0ct. and 30 Oct. I9I6). M. DE LA VAlLÉE-Poussis obtains, by methods quite unlike those which we use here, a considerable part of the results of section 4 (I8 Nov. I9I6).

\section{Erratum}

G. H. HARDY and J. E. LITTLEwood, 'Some problems of Diophantine Approximation', II, Acta Mathematica, vol. 37, p. 23I, line I:

$$
\text { for } o\left\{\sqrt{\frac{I}{I-r}}\right\} \text { read o }\left\{\sqrt{\left.\frac{I}{I-r}\right\}}\right. \text {. }
$$

